\title{
Rapid Detection of Porcine Reproductive and Respiratory Syndrome Virus by Reverse Transcription Loop-Mediated Isothermal Amplification
}

\author{
Chen Qin ${ }^{\mathrm{a}} \quad$ Li Jian $^{\mathrm{b}} \quad$ Fang Xue-En ${ }^{\mathrm{a}} \quad$ Xiong Wei $^{\mathrm{b}}$ \\ ${ }^{a}$ School of Life Science, Shanghai University, and ${ }^{b}$ Shanghai Entry-Exit Inspection and Quarantine Bureau, \\ Shanghai, People's Republic of China
}

\section{Key Words}

Porcine reproductive and respiratory syndrome virus •

Reverse transcription loop-mediated isothermal

amplification • Detection

\begin{abstract}
The fragment of the membrane protein $M$ gene with high conservation and specificity of porcine reproductive and respiratory syndrome virus (PRRSV) was chosen to be the target region, according to which six special primers were designed successfully. Reverse transcription loop-mediated isothermal amplification (RT-LAMP) was applied to detect the PRRSV by incubation at $65^{\circ}$ for only 45 min with the ladder-like pattern of bands from 182 bp on the agarose gel, and the product specificity was confirmed by $R$ sal. The result of RT-LAMP could also be visualized directly with the naked eye by adding the intercalating dye Picogreen ${ }^{\circledR}$. The RTLAMP was identified to detect only the PRRSV in all viruses tested, which demonstrated the high specificity. By using various sample dilutions as templates, the sensitivity of RTLAMP was found to be 100-fold higher than that of RT-PCR and could be comparable to the fluorescence quantitative RT-PCR. A comparison was obtained by the RT-LAMP and PCR assays using 20 clinical samples. Finally, a rapid, convenient and reliable PRRSV detection system was developed using the RT-LAMP.

Copyright $\odot 2009$ S. Karger AG, Basel
\end{abstract}

\section{Introduction}

Porcine reproductive and respiratory syndrome virus (PRRSV) is an enveloped, single-stranded RNA virus, which could cause reproductive failure of sows and respiratory problems of piglets and growing pigs, leading to a serious financial loss to the whole pig industry [1]. Conventional PRRSV detection is performed by direct virus isolation [2, 3], ELISA [4, 5] or RT-PCR [6-8]. However, the shortcomings such as precise instrument requirement, elaborate result analysis demand, high cost and long detection time prevent these methods from being widely used.

Loop-mediated isothermal amplification (LAMP) is a novel nucleic acid amplification method, which amplifies DNA with high specificity, sensitivity and rapidity under isothermal condition [9]. LAMP can also amplify RNA with high efficiency by directly adding the reverse transcriptase to the reaction mixture, which is termed the reverse transcription (RT)-LAMP. It has been used widely for RNA virus detection, such as foot-and-mouth disease virus [10], Taura syndrome virus [11], infectious hypodermal and hematopoietic necrosis virus [12], severe acute respiratory syndrome coronavirus [13] and H5N1 avian influenza virus [14]. In this study, RT-LAMP was applied to detect PRRSV and its specificity and sensitivity were assessed.

\section{KARGER}

Fax +4161306 1234 E-Mail karger@karger.ch www.karger.com
Chen Qin

School of Life Science, Shanghai University

Shanghai (People's Republic of China)

Tel. +862166134332

E-Mail chenqincc@yahoo.com.cn 


\section{Materials and Methods}

\section{Samples}

Porcine reproductive and respiratory syndrome virus (strain SD1, strain HUB1, strain CH-1a, strain JXwn06, BJ-4, HN1), swine transmissible gastroenteritis coronavirus (TGEV), swine fever virus (SFV), porcine parvovirus (PPV), pseudorabies virus (PRV) and foot-and-mouth disease virus (FMDV) were all derived from their passages in cell culture which were provided by Shanghai Entry-Exit Inspection and Quarantine Bureau (SHCIQ). The clinical samples, including porcine blood and lung, were collected from a suspect case of PRRSV.

\section{LAMP Primer Designing}

Nucleic acid sequences of different PRRSV strains/isolates and other similar viruses were obtained from GenBank, and the homology was analyzed using the Vector NTI. The conserved fragment with high homology was chosen to be the target region which was used to design the PRRSV RT-LAMP primers by the Primer Explorer V3 software (http://primerexplorer.jp/e/).

\section{RNA Extraction}

Total genomic RNA was extracted using the Trizol kit (Invitrogen, Germany). After elution in $20 \mu$ l nuclease-free $\mathrm{H}_{2} \mathrm{O}$, the RNA sample was stored at $-70^{\circ}$ until used.

\section{RT-LAMP}

The RT-LAMP reaction was carried out in a volume of $25 \mu \mathrm{l}$ containing $1 \times$ ThermoPol buffer (New England Biolabs, USA), $6.0 \mathrm{mM} \mathrm{MgSO}_{4}, 0.8 \mathrm{M}$ betaine (Sigma, Germany), 1.0 mM dNTPs, $0.2 \mu \mathrm{M}$ each of outer primer, $1.6 \mu \mathrm{M}$ each of inner primer and 0.4 $\mu \mathrm{M}$ each of loop primer, $5 \mathrm{U}$ AMV reverse transcriptase, $8 \mathrm{U}$ of Bst polymerase (Large Fragment; New England Biolabs, USA) with $2 \mu$ l total RNA as template. The amplification was performed at $65^{\circ}$ in a laboratory water bath (Kangle, China, 25-99, $\pm 0.3^{\circ}$ ) for $45 \mathrm{~min}$.

The reaction mixture was digested with $R s a$ I to confirm the specificity of PRRSV RT-LAMP products. The amplification/digestion products were separated by the agarose gel electrophoresis and visualized by staining with ethidium bromide. The result of RT-LAMP could also be visualized directly with the naked eye according to the green color produced by adding $10 \mu$ intercalating dye Picogreen ${ }^{\circledR}$ (Invitrogen, USA).

\section{Specificity of RT-LAMP}

The specificity of RT-LAMP was examined by the use of RNA (or DNA) extracted from six different PRRSV strains and five other viruses known to be related genetically to PRRSV or to cause similar clinical signals in pig.

Sensitivity of RT-LAMP and Comparison with Other RT-PCRs

The sensitivity of RT-LAMP was evaluated and compared with RT-PCR (PRRSV RT-PCR diagnostic kit; Suoaobio, China) and fluorescence quantitative RT-PCR (PRRSV fluorescence quantitative RT-PCR diagnostic kit; Suoaobio, China) using various PRRSV RNA dilutions $\left(10^{-1}\right.$ to $\left.10^{-5}\right)$ as templates.

Evaluation of the RT-LAMP Assay Using Clinical Samples

The evaluation of the RT-LAMP assay was performed using RNA extracted from clinical samples with RT-PCR and fluorescence PCR running in parallel.

Detection of Porcine Reproductive and Respiratory Syndrome Virus by RT-LAMP
Table 1. Homology of the target sequence in different PRRSV strains/isolates and other similar viruses

\begin{tabular}{|c|c|c|}
\hline Viruses & $\begin{array}{l}\text { GenBank ac- } \\
\text { cession No. }\end{array}$ & Identity \\
\hline PRRSV RespPRRS MLV & AF066183 & 100 \\
\hline PRRSV isolate MLV RespPRRS/Repro & AF159149 & 100 \\
\hline PRRSV isolate PA8 & AF176348 & 100 \\
\hline PRRSV isolate NVSL 97-7985 IA 1-4-2 & AF325691 & 97.8 \\
\hline PRRSV virus BJ-4 & AF331831 & 100 \\
\hline PRRSV isolate P129 & AF494042 & 98.4 \\
\hline PRRSV strain SP & AF184212 & 96.2 \\
\hline PRRSV strain $\mathrm{CH}-1 \mathrm{a}$ & AY032626 & 97.3 \\
\hline PRRSV isolate VR-2332 & AY150564 & 100.0 \\
\hline PRRSV strain JA142 & AY424271 & 96.7 \\
\hline PRRSV HN1 & AY457635 & 99.5 \\
\hline PRRSV strain NVSL 97-7895 & AY545985 & 96.7 \\
\hline PRRSV strain PL97-1 & AY585241 & 100 \\
\hline PRRSV strain PL97-1/LP1 & AY612613 & 100 \\
\hline PRRSV isolate MN184A & DQ176019 & 96.7 \\
\hline PRRSV isolate MN184B & DQ176020 & 96.7 \\
\hline PRRSV isolate $\mathrm{S} 1$ & DQ459471 & 100.0 \\
\hline PRRSV isolate LMY & DQ473474 & 98.9 \\
\hline PRRSV strain PrimePac & DQ779791 & 96.2 \\
\hline PRRSV isolate Ingelvac ATP & DQ988080 & 96.2 \\
\hline PRRSV strain HUB1 & EF075945 & 97.3 \\
\hline PRRSV strain JXA1 & EF112445 & 97.8 \\
\hline PRRSV strain HUB2 & EF112446 & 97.3 \\
\hline PRRSV strain HEB1 & EF112447 & 97.8 \\
\hline PRRSV isolate CC-1 & EF153486 & 100 \\
\hline PRRSV clone pJX143 & EF488048 & 97.8 \\
\hline PRRSV isolate MN184C & EF488739 & 96.7 \\
\hline PRRSV strain HuN & EF517962 & 97.8 \\
\hline PRRSV strain HUN4 & EF635006 & 97.8 \\
\hline PRRSV strain JXwn06 & EF641008 & 97.8 \\
\hline PRRSV strain NX06 & EU097706 & 97.8 \\
\hline PRRSV strain BJsy06 & EU097707 & 97.8 \\
\hline PRRSV strain SHH & EU106888 & 97.8 \\
\hline PRRSV strain LN & EU109502 & 97.8 \\
\hline PRRSV isolate SY0608 & EU144079 & 97.8 \\
\hline PRRSV strain WUH1 & EU187484 & 97.8 \\
\hline PRRSV strain Jiangxi-3 & EU200961 & 97.8 \\
\hline PRRSV strain Henan-1 & EU200962 & 97.8 \\
\hline PRRSV isolate HPBEDV & EU236259 & 97.8 \\
\hline PRRSV strain rV68 & EU360128 & 100 \\
\hline PRRSV strain rV63 & EU360129 & 100 \\
\hline PRRSV strain HB-1/3.9 & EU360130 & 97.8 \\
\hline SFV & DQ127910 & 49.5 \\
\hline FMDV & NC004004 & 46.3 \\
\hline PCV & NC-001792 & 47.2 \\
\hline TGEV & NC002306 & 49.7 \\
\hline PPV & NC001718 & 48.7 \\
\hline SDV & EU151461 & 48.4 \\
\hline
\end{tabular}


Fig. 1. The conserved fragment in the membrane protein $\mathrm{M}$ gene.
5'GGGAGCAGTAGTTGCACTCCTTTGGGGGGTGTACTCAGCCATAGAAACCTGGAAATT CATCACCTCCAGATGCCGTTTGTGCTTGCTAGGCCGCAAGTACATTCTGGCCCCTGCCC ACCACGTTGAAAGTGCCGCAGGCTTTCATCCGATTGCGGCAAATGATAACCACGCATTT GTCGTCC3'

Table 2. PRRSV RT-LAMP primers

\begin{tabular}{llll}
\hline $\begin{array}{l}\text { Primer } \\
\text { name }\end{array}$ & Type & $\begin{array}{l}\text { Length } \\
\text { bp }\end{array}$ & Sequence $\left(5^{\prime}\right.$ to $\left.3^{\prime}\right)$ \\
\hline F3 & forward outer & 19 & GGGAGCAGTAGTTGCACTC \\
B3 & reverse outer & 20 & GGACGACAAATGCGTGGTTA \\
FIP & forward inner & 39 & GCACAAACGGCATCTGGAGGTTTGGGGGGTGTACTCAGC \\
BIP & reverse inner & 40 & TAGGCCGCAAGTACATTCTGGCTTTGCCGCAATCGGATGA \\
LF & forward loop & 21 & ATGAATTTCCAGGTTTCTATG \\
LB & reverse loop & 20 & CACGTTGAAAGTGCCGCAGG \\
\hline
\end{tabular}

\section{Results}

\section{Designing the RT-LAMP Primers}

The nucleic acid sequence homology of different PRRSV strains/isolates and other similar viruses were analyzed by Vector NTI to find a conserved fragment. The results are shown in table 1 . The fragment (fig. 1) was located in the membrane protein $\mathrm{M}$ gene with high conservation and specificity. The identity of the region was higher than $96 \%$ among 32 PRRSV strains/isolates but lower than 50\% among 6 other similar viruses. In addition, of all the PRRSV strains/isolates analyzed, 6 strains/isolates revealed the $100 \%$ homology, accounting for $18.7 \%$. The PRRSV RT-LAMP primers were designed according to the conserved sequence by the Primer Designer V3 (table 2).

\section{PRRSV RT-LAMP}

RT-LAMP was used to detect the PRRSV with the special primers above and the specificity of the product was confirmed by RsaI. Amplification/digestion products were analyzed by agarose gel electrophoresis. As shown in figure $2 \mathrm{a}$ (lanes 1,3 ), the positive sample was amplified at $65^{\circ}$ with a ladder-like pattern of bands from $182 \mathrm{bp}$ on the gel, while the negative sample showed no bands. After digestion with RsaI, products of a predictable size of the 84bp motif were resolved on the gel (fig. 2a; lane 2). The positive reaction could also be visualized directly with the naked eye according to the green color produced by Picogreen (fig. 2b; lanes 1', 3').

\section{Specificity of RT-LAMP}

The six different PRRSV strains and five other viruses were investigated to evaluate the specificity of the RTLAMP for PRRSV detection. The results showed that only the PRRSV amplified while no amplification was performed in all other tested viruses (fig. 3).

\section{Sensitivity of RT-LAMP and Comparison with Other RT-PCRs}

The sensitivity of RT-LAMP was evaluated and compared with RT-PCR and fluorescence quantitative RTPCR tests using various PRRSV RNA dilutions as templates. As shown in figure 4, RT-LAMP was able to detect a template at $10^{-4}$ dilution, whereas the RT-PCR and fluorescence quantitative RT-PCR could detect a template at $10^{-2}$ and $10^{-4}$ dilutions, respectively. Therefore, the sensitivity of RT-LAMP was 100-fold higher than that of RTPCR and could be comparable to the fluorescence quantitative RT-PCR.

\section{Evaluation of the RT-LAMP Assay Using Clinical Samples}

The evaluation of the RT-LAMP was performed using RNA extracted from 20 clinical samples with RT-PCR and fluorescence PCR running in parallel. The results of RTLAMP correlated well with the other two tests (table 3). 


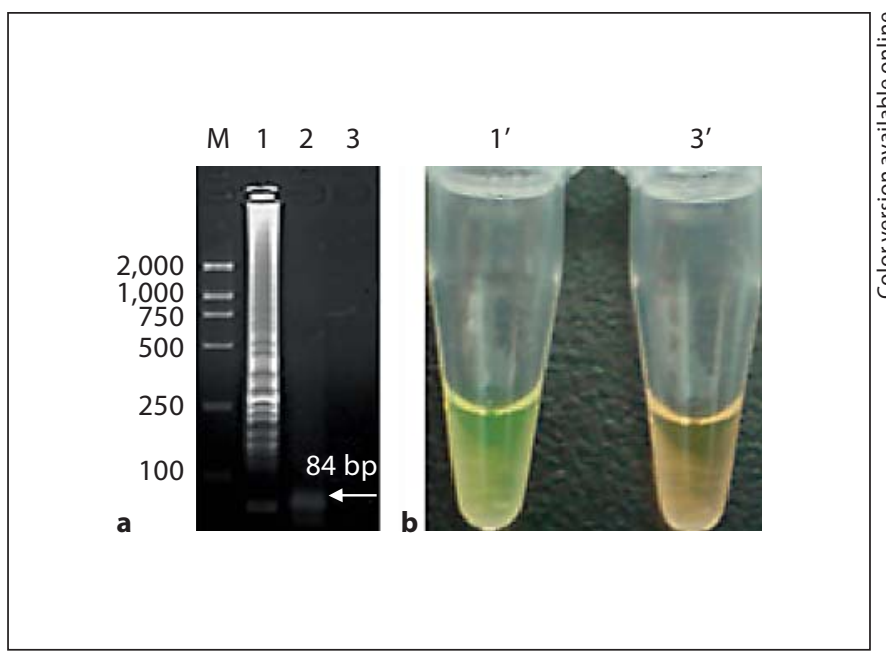

Fig. 2. PRRSV RT-LAMP. a Agarose gel electrophoresis. b Visual inspection of RT-LAMP. $1,1^{\prime}=$ Positive reaction; 2 = products digested with $R s a \mathrm{I} ; 3,3^{\prime}=$ negative reaction.

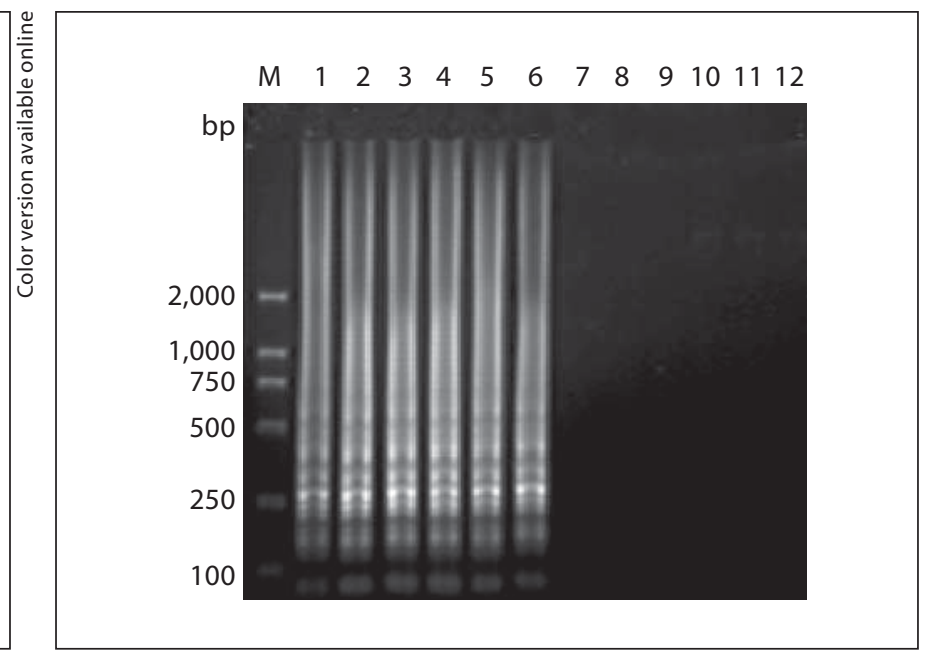

Fig. 3. Specificity of RT-LAMP. 1-11 = PRRSV (strain SD1, strain HUB1, strain CH-1a, strain JXwn06, BJ-4, HN1), TGEV, SFV, PPV, PRV and FMDV, respectively; 12 = negative control.
Fig. 4. Sensitivity of RT-LAMP and comparison with other RT-PCRs. a RT-LAMP. b RT-PCR. c Fluorescence quantitative RTPCR. $1-5=$ RNA sample at $10^{-1}, 10^{-2} \ldots$ $10^{-5}$ dilutions, respectively; $6=$ negative control.

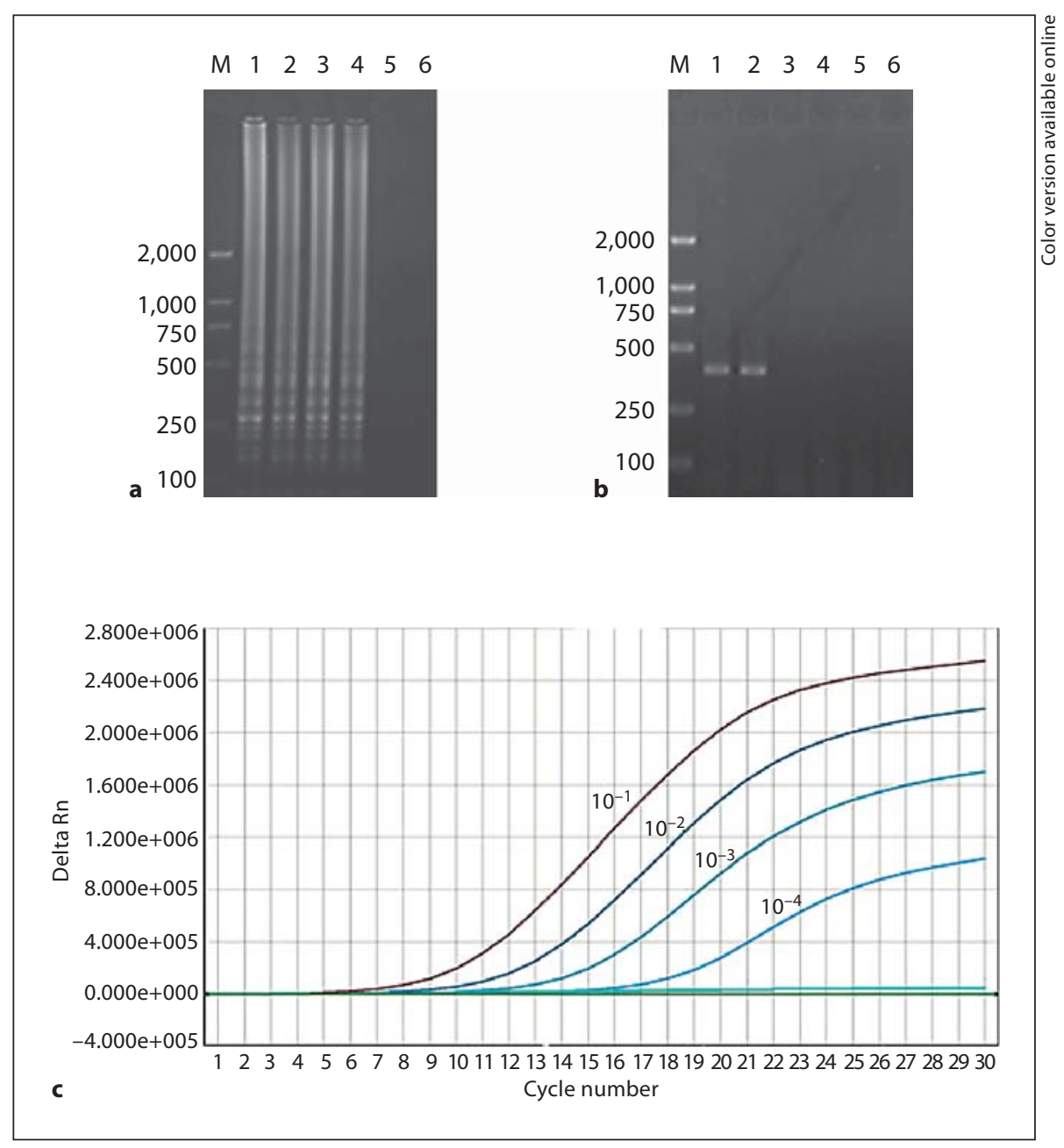

Intervirology 2009;52:86-91 
Table 3. Detection results of RT-LAMP, RT-PCR and fluorescence PCR with field samples

\begin{tabular}{|c|c|c|c|c|}
\hline \multicolumn{2}{|c|}{ Sample } & \multirow{2}{*}{$\begin{array}{l}\text { RT-LAMP } \\
-\end{array}$} & \multirow{2}{*}{$\begin{array}{l}\text { RT-PCR } \\
-\end{array}$} & \multirow{2}{*}{$\begin{array}{l}\text { Fluorescence } \\
\text { PCR } \\
-\end{array}$} \\
\hline Blood & 1 & & & \\
\hline & 2 & - & - & - \\
\hline & 3 & - & - & - \\
\hline & 4 & + & + & + \\
\hline & 5 & - & - & - \\
\hline & 6 & - & - & - \\
\hline & 7 & + & + & + \\
\hline & 8 & - & - & - \\
\hline & 9 & - & - & - \\
\hline & 10 & - & - & - \\
\hline \multirow[t]{10}{*}{ Lung } & 1 & + & + & + \\
\hline & 2 & - & - & - \\
\hline & 3 & - & - & - \\
\hline & 4 & + & + & + \\
\hline & 5 & - & - & - \\
\hline & 6 & - & - & - \\
\hline & 7 & - & - & - \\
\hline & 8 & - & - & - \\
\hline & 9 & - & - & - \\
\hline & 10 & + & + & + \\
\hline
\end{tabular}

\section{Discussion}

It is very important to find a conserved nucleic acid fragment and design special RT-LAMP primers, as PRRSV strains/isolates exhibit a significant heterogeneity at the nucleotide level $[1,15-17]$. In this study, the nucleic acid sequence homology of 32 PRRSV strains/isolates and 6 other similar viruses available in GenBank was analyzed by Vector NTI. The most conserved fragment of $182 \mathrm{bp}$ was found in the membrane protein $\mathrm{M}$ gene which showed high homology among different PRRSV strains/isolates (more than 96\%) and low homology among other similar viruses (less than 50\%). The PRRSV RT-LAMP primers targeting the conserved sequence were designed successfully by the Primer Designer V3 software after screening according to the end stability of the primers.

The target region was amplified successfully by the RT-LAMP diagnostic protocol with a characteristic ladder-like pattern of bands on the gel. This is because the final products of RT-LAMP are a mixture of stem-loop DNAs with various stem lengths and cauliflower-like structures with multiple loops formed by annealing be- tween alternately inverted repeats of the target sequence in the same strand [9]. After digestion with RsaI, the 84bp motif was resolved on the gel as expected, which demonstrated the specific structure of amplification products. RT-LAMP result could also be analyzed by directly adding the intercalating dye Picogreen ${ }^{\circledR}$. Positive amplification exhibited a green color, while a negative reaction corresponded to an orange color [10], which would facilitate the application of RT-LAMP, especially as a field test.

Five other viruses were used in the study to confirm the specificity of RT-LAMP. The results showed no amplification in all viruses tested, which makes the RTLAMP more accurate and reliable for PRRSV detection. The high specificity of LAMP is most probably attributable to recognition of the target sequence by six independent sequences in the initial stage and by four independent sequences during the second reaction stage [9]. The sensitivity of RT-LAMP was evaluated using various PRRSV RNA dilutions as templates. The assay demonstrated almost equivalent sensitivity to the fluorescence quantitative RT-PCR and 100-fold higher than the RT-PCR, which indicates that the RT-LAMP is a more powerful diagnostic tool to detect PRRSV in low copy numbers. A good correlation was obtained by the RTLAMP, RT-PCR and fluorescence PCR, which demonstrated the accuracy and reliability of the RT-LAMP in the PRRSV detection. Furthermore, the rapid detection and simple operation of the PRRSV RT-LAMP developed represent advantages. The only equipment required for the reaction is a water bath or heat block. The assay developed is a faster detection method for the PRRSV detection, only taking about $45 \mathrm{~min}$, which means the whole diagnosis including RNA extraction, amplification and product detection could be completed within $1.5 \mathrm{~h}$ after arrival of the samples. It is anticipated that with the advantages of specificity, sensitivity, reliability, rapidity and easy manipulation, LAMP will be a powerful molecular tool for the PRRSV detection in practice. 


\section{References}

1 Benfield DA, Collins JE, Dee SA, Hallbur PG, Joo HS, Lager KM, Mengeling WL, Murtaugh MP, Rossow KD, Stevenson GW, Zimmerman JJ: Porcine reproductive and respiratory syndrome; in Straw BE, D'Allaire S, Mengeling WL, Taylor DJ: Diseases of Swine, ed 8. Ames, Iowa State University Press, 1999, pp 201-232.

-2 Collins JE, Benfield DA, Christianson WT, Harris L, Hennings JC, Shaw DP, Goyal SM, McCullough S, Morrison RB, Joo HS, Gorcyca D, Chladek D: Isolation of swine infertility and respiratory syndrome virus (isolate ATCC VR-2332) in North America and experimental reproduction of the disease in gnotobiotic pigs. J Vet Diagn Invest 1992;4: 117-126.

-3 Botner A, Neilsen J, Bille-Hansen V: Isolation of porcine reproductive and respiratory syndrome (PRRS) virus in a Danish swine herd and experimental infection of pregnant gilts with the virus. Vet Microbiol 1994;40: 351-360.

-4 Denac H, Moser C, Tratschin JD, Hofmann MA: An indirect ELISA for the detection of antibodies against porcine reproductive and respiratory syndrome virus using recombinant nucleocapsid protein as antigen. J Virol Methods 1997;65:169-181.
5 Albina E, Leforban Y, Baron T, Plana DJ, Vannier P: An enzyme linked immunosorbent assay (ELISA) for the detection of antibodies to the porcine reproductive and respiratory syndrome (PRRS) virus. Ann Rech Vet 1992;23:167-176.

6 Van Woensel P, Van der Wouw J, Visser N: Detection of porcine reproductive and respiratory syndrome virus by polymerase chain reaction. J Virol Methods 1994;47:273-278.

7 Christopher HJ, Nelson EA, Nelson JK, Hines RJ, Swenson SL, Hill HT, Zimmerman JJ, Katz JB, Yaeger MJ, Chase CCL, Benfield DA: Detection of porcine reproductive and respiratory syndrome virus in boar semen by PCR. J Clin Microbiol 1995;33:1730-1734.

-8 Kono Y, Kanno T, Shimzu M, Yamada S, Ohashi S, Nakamine M, Shirai J: Nested PCR for the detection and typing of porcine reproductive and respiratory syndrome (PRRS) virus in pigs. J Vet Med Sci 1996;58: 941-946.

-9 Notomi T, Okayama H, Masubuchi H: Loopmediated isothermal amplification of DNA. Nucleic Acids Res 2000;28:e63.

10 Dukes JP, King DP, Alexandersen S: Novel reverse transcription loop-mediated isothermal amplification for rapid detection of foot-and-mouth disease virus. Arch Virol 2006;151:1093-1106.

11 Kiatpathomchai W, Jareonram W, Jitrapakdee S: Rapid and sensitive detection of Taura syndrome virus by reverse transcription loop-mediated isothermal amplification. J Virol Methods 2007;146:125-128.
12 Zhao FS, Hu CQ, Ren CH: Sensitive and rapid detection of infectious hypodermal and hematopoietic necrosis virus (IHHNV) in shrimps by loop-mediated isothermal amplification. J Virol Methods 2006;131:4146.

13 Hong TC, Mai QL, Cuong DV: Development and evaluation of a novel loop-mediated isothermal amplification method for rapid detection of severe acute respiratory syndrome coronavirus. J Clin Microbiol 2004;4:19561961.

14 Imaia M, Ninomiy A, Minekaw H: Rapid diagnosis of $\mathrm{H} 5 \mathrm{~N} 1$ avian influenza virus infection by newly developed influenza $\mathrm{H} 5$ hemagglutinin gene-specific loop-mediated isothermal amplification method. J Virol Methods 2007;141:173-180.

15 Conzelmann KK, Visser N, Van WP, Thiel $\mathrm{HJ}$ : Molecular characterization of porcine reproductive and respiratory syndrome virus, a member of the arterivirus group. Virology 1993;193:329-339.

16 Christianson WT, Joo HS: Porcine reproductive and respiratory syndrome. Swine Health Prod 1994;2:10-28.

17 Oleksiewicz MB, Bøtner A, Madsen KG, Storgaard T: Sensitive detection and typing of porcine reproductive and respiratory syndrome virus by RT-PCR amplification of whole viral gene. Vet Microbiol 1998;64:722. 